\title{
Vaginal dehydroepiandrosterone compared to other methods of treating vaginal and vulvar atrophy associated with menopause
}

\author{
Wojciech Pięta, Roman Smolarczyk \\ Department of Gynecological Endocrinology, Medical University of Warsaw, Poland
}

\begin{abstract}
During the menopause, a fall in estrogen levels often leads to many unfavorable symptoms, including changes in the vascular and urogenital systems, in mood, and sleep. The symptoms of vulvovaginal atrophy are especially troublesome for menopausal women. These symptoms not only disturb the sexual sphere, but also functioning at work and in the family. Based on the literature, a review of contemporary methods of management in the case of symptoms of vulvar atrophy in menopausal women has been performed. The current methods of treating vulvovaginal atrophy in menopausal women are described. The pharmacology of the available dehydroepiandrosterone (DHEA) preparations, both oral and vaginal, was briefly analyzed. Own experiences of using DHEA are presented. Vaginal DHEA has been found to be an effective and safe treatment in menopausal women with symptoms of vaginal atrophy.
\end{abstract}

Key words: DHEA, menopause, vulvovaginal atrophy.

\section{Introduction}

Menopause is associated with physiological and psychological changes that influence sexuality. During menopause, the primary biological change is decrease in circulating estrogen levels. Estrogen deficiency initially accounts for altered bleeding and diminished vaginal lubrication. Continual estrogen loss often leads to numerous signs and symptoms, including changes in the vascular and urogenital systems. alterations in mood, sleep, and cognitive functioning are common as well. These changes may contribute to lower selfesteem, poorer self-image, and diminished sexual responsiveness and sexual desire. The problems in sexual functioning related to estrogen deficiency can be treated with hormone therapy that includes estrogens alone and estrogens combined with androgens. Vaginal lubricants and moisturizers also may be useful in ameliorating postmenopausal sexual complaints [1]. The symptoms associated with vaginal atrophy are particularly troublesome for menopausal women.

\section{Estrogen}

The most common treatment for vaginal atrophy in menopausal women is the topical use of estrogens 17- $\beta$ estradiol or estriol [2]. Currently, numerous studies can be found in the literature on alternative treatments for this disease and its symptoms.

\section{Tissue selective estrogen complex}

A tissue selective estrogen complex (TSEC) pairs a selective estrogen receptor modulator (SERM) with estrogen(s). Clinical studies suggest that the therapeutic effect of this combination of drugs on vaginal atrophy is estrogen dose dependent [3].

\section{Selective estrogen receptor modulators}

\section{Ospemifene}

Ospemifene (deaminohydroxytoremifene) is a selective estrogen receptor modulator (SERM) acting similarly to an estrogen on the vaginal epithelium. Di Donato's studies have shown the use of ospemifene in a dose of $60 \mathrm{mg}$ is associated with a significant improvement in the morphological and physiological characteristics of the vaginal mucosa, which correlate with the symptoms associated with vulvovaginal atrophy after menopause [4].

\section{Lasofoxifene}

Lasofoxifene is a nonsteroidal selective estrogen receptor modulator (SERM). Lasofoxifene $0.25 \mathrm{mg}$ and $0.5 \mathrm{mg}$ has demonstrated an effect in treating vulvovaginal atrophy. Over 12-weeks treatment compared to baseline Lasofoxifene significantly reduced the most bothersome moderate to severe vulvovaginal atrophy symptom [5]. 


\section{Oxytocin}

Oxytocin (Oxt) is a peptide hormone and neuropeptide. It is normally produced in the hypothalamus and released by the posterior pituitary. This year's research showed that eight- week intervention with oxytocin vaginal gel (400 IU) could significantly improve the vaginal maturation index, subjective symptoms of vaginal atrophy and reduce the $\mathrm{PH}$ of the vagina. Using this medication in women who have a contraindication for hormone therapy is recommended [6].

\section{Genistein}

Genistein $\left(\mathrm{C}_{15} \mathrm{H}_{10} \mathrm{O}_{5}\right)$ is a naturally occurring compound that structurally belongs to a class of compounds known as isoflavones. It is described as an angiogenesis inhibitor and a phytoestrogen.

Genistein improved genital symptoms, colposcopical features and maturation value. Genistein showed slight decrease in flow cytometric analysis of DNA ploidy, with a normalization of the aneuploid content present in some cases that could represent an additional application of intravaginal phytoestrogen therapy, providing an alternative therapy of vaginal atrophy in postmenopausal patients [7].

\section{Fractionated laser}

The laser treatment for vaginal atrophy involves placing a laser head in the vagina and takes about 5-10 minutes. During this time, energy in the form of radiation is delivered to the area of the vaginal opening and the vaginal canal. First placebo-controlled RCT reports no statistically significant differences in any parameter (vaginal histopathology and cytology, self-reported symptom severity by visual analogue scale of most bothersome symptom, impact on livelihood, quality of life and sexual function) between placebo and intervention groups using fractionated $\mathrm{CO}_{2}$ laser for postmenopausal vaginal atrophy symptoms [8].

\section{DHEA}

\section{Pharmacology}

Dehydroepiandrosterone (DHEA) is an endogenous steroidal hormone secreted by Zona reticularis of the adrenal cortex with a characteristic age-related pattern of secretion. It is present in higher con-centration in plasma than any other steroid hormone. Adrenal production of DHEA begins during puberty, peak at the age of 20 years and decline with ageing beginning at the age of 25 years[. This hormone progressively declines at the rate of $2 \%$ per year. It serves as an indirect precursor for male and female reproductive hormone i.e. estrogen and testosterone and various other steroidal hormones [9].
DHEA (3ß-hydroxyandrost-5-en-17-one) shares the same basic four-linked cyclopentanophenanthrene ring structure with other steroids. Its classification as anandrogen relates to the presence of 19 carbon (C) atoms. The hydroxyl group (alcoholmoiety)at posi-tion $3 \beta$ on the DHEA molecule allows for its conjugation with sulfate at this location. DHEA is sulfated to form DHEAS by sulfo-transferase (SULT2A1) involving the co-substrate 3'-phospho-adenosine-5'-phos-phosulfate (PAPS). Conversely, sulfatase- 1 and -2 converts DHEAS to DHEA. $\mathrm{DHEA}(\mathrm{S})$ production is largely dictated by the relative tissue distribution of the steroid enzymes. High levels of the enzyme SULT2A1are found in the adrenal gland and liver. Sulfated steroids have long been considered as inactive hydrophilic metabolites destinedf orurinary excretion, but identification of specific uptake carriers for sulfatedsteroidhormones, (e.g.SOAT,sodium-de -pendentorganicaniontransporter), and the intracellular presence of steroid sulfatases leads to the conclusion that sulfated steroids have a role as intracrineregulators within specific target cell [10].

In women during the reproductive years in addition to the sex steroids testosterone and estrogen, the adrenal glands and ovaries produce DHEA. Its sulphated form dehydroepiandrosterone sulphate (DHEAS) is primarily synthesized by the adrenals, has a longer half-life and provides a stable pool of DHEA. DHEA is converted to estrogen and testosterone in peripheral tissues such as brain, bone, breast and ovaries. With increasing age the adrenals are the primary source of both DHEA and DHEAS and therefore indirectly the main source of estrogen and testosterone [11].

DHEA may be best understood as an androgenic precursor whose biological actions are effected through the production of testosterone and dihydrotestosterone. DHEA mediates its action via multiple signaling pathways involving specific membrane receptors and via transformation into androgen and estrogen derivatives (e.g., androgens, estrogens, $7 \alpha$ and $7 \beta$ DHEA, and $7 \alpha$ and $7 \beta$ epiandrosterone derivatives) acting through their specific receptors. These pathways include: nitric oxide synthase activation, modulation of $\gamma$-amino butyric acid receptors, $\mathrm{N}$-methyl $\mathrm{D}$-aspartate, receptors sigma receptors (Sigma-1), differential expression of inflammatory factors, adhesion molecules and reactive oxygen species, among others [12].

\section{Oral administration}

In case oral administration, the data suggest that DHEA could counteract the effect of aging by acting simultaneously on both skin compartments namely the dermis, by stimulating collagen

biosynthesis and deposition, and in the epidermis by modulating keratinocyte proliferation and differen- 
tiation. Some of the effects observed are reminiscent of wound healing, thus suggesting that

DHEA treatment could potentially lead to apparent skin rejuvenation. It is recognized that the skin undergoes regressive changes after menopause and that these changes a remainly related to a loss of skin collagen content. The present data suggest that DHEA could exert anti-aging effects in the skin, secondary to DHEA-induced changes in the structural organization of the dermis [13].

Transformation of topical DHEA in postmenopausal women is preferentially into androgens rather than into estrogens. On the other hand, the present data indicate that serum DHEA measurements following DHEA supplementation in postmenopausal women are an overestimate of the formation of active androgens and estrogens and suggest a decreased efficiency of transformation of DHEA into androgens and estrogens with aging [14].

Leblanc's study provides detailed information on the effect of a single $50 \mathrm{mg}$ oral dose of DHEA on circulating estrogens as well as androgens and their metabolites over $10 \mathrm{~h}$ in adult ovariectomised (OVX) Cynomolgus monkeys. Serum DHEA, DHEA-S, testosterone (Testo) and androstenedione (4-dione) concentrations increased rapidly with a maximal value at approximately $1 \mathrm{~h}$ after DHEA administration followed by a 60-80\% decrease during the next 2-6 h. An important sulfatation of DHEA occurs through first hepatic pass, thus, leading to a marked increase in serum DHEA-S. Serum androst-5-ene-3,17-diol and androsterone glucuronide (ADT-G) levels remained elevated on a plateau for $6 \mathrm{~h}$. Androstan-3,17-diol-glucuronide, estradiol and estrone levels remained unchanged. The present data indicate the predominant transformation of the adrenal precursor DHEA into active androgens in peripheral tissues and support the importance of measurement of circulating glucuronide derivatives as index of peripheral or intracrine androgen formation and action [7, 15].

\section{Vaginal administration}

Labrie's research has shown that the use of even high doses of vaginal DHEA in postmenopausal women raises the level of this hormone to the levels found in normal premenopausal women. Similar observations were made for serum androstenedione, estrone, estrone-sulfate and DHEA-sulfate. The data show that the intravaginal administration of DHEA permits to rapidly achieve the local beneficial effects against vaginal atrophy without significant changes in serum estrogens, thus avoiding the increased risk of breast cancer associated with the current intravaginal or systemic estrogenic formulations [16].

\section{Clinical efficacy (literature review)}

After reviewing the literature, Archer concluded that vaginal DHEA administered daily over a 12-week period was found to improve symptoms and physical findings of vulvovaginal atrophy in postmenopausal women compared to baseline and placebo using objective criteria. Dyspareunia and libido, two factors associated with quality of life,were significantly improved with DHEA treatment. Thes echanges took place withouta biologically significant increase in circulating estradiol levels indicating that the DHEA effectis via intracrinology, or intracellular conversion of DHEA to estradiol. Hormonal treatment of vulvovaginal atrophy without elevatin gcirculating Estradiol levels isi mportant for the healthcare provider and consumer.Thus intravaginal DHEA isa viable treatment option in postmenopausal women with vulvovaginal atrophy [17].

Labrie in his research proved that standard 12-week taking $0.5 \%$ DHEA caused a $45.9 \pm 5.31$ ( $p<0.0001$ versus placebo) decrease in the $\%$ of parabasal cells, a $6.8 \pm 1.29 \%$ ( $p<0.0001)$ increase in superficial cells, a $1.3 \pm 0.13$ unit $(p<0.0001)$ decrease in vaginal $\mathrm{pH}$ and a $1.5 \pm 0.14$ score unit $(p<0.0001)$ decrease in the severity of the most bothersome symptom. Similar changes were seen on vaginal secretions, color, epithelial surface thickness and epithelial integrity [18].

Debra et al. studied a group of women (hundred forty-five) who had been treated for breast cancer with symptoms of vaginal atrophy. Some of the participants were treated with tamoxifen or an aromatose inhibitor. Women were randomized to 3.25 versus $6.5 \mathrm{mg} /$ day of DHEA versus a plain moisturizer control. DHEAS and testosterone increased significantly in the group of women taking the DHEA vaginally compared to the group of women taking the moisturizer. Estradiol only increased in the $6.5 \mathrm{mg} /$ day DHEA group. Maturation of vaginal cells was $100 \%$ ( $3.25 \mathrm{mg} /$ day), $86 \%$ (6.5 mg/day), and $64 \%$ (moisturizer); $\mathrm{pH}$ decreased more in DHEA arms. The authors conclude that the use of vaginal DHEA in women after breast cancer treatment resulted in increased hormone concentrations, though still in the lowest half or quartile of the postmenopausal range, and provided more favorable effects on vaginal cytology, compared to the moisturizer [19].

In a prospective, randomized, double-blind, placebocontrolled phase III clinical trial, Labrie et al. investigated the beneficial effect of vaginal dehydroepiandrosterone $-0.50 \%$ DHEA $(6.5 \mathrm{mg})$. The treatment population included 157 (placebo) and 325 (DHEA-treated) women. The investigators concluded that the strictly local effect of Prasterone is consistent with the lack of significant drug-related adverse events, indicating a high benefitrisk ratio of this treatment based on a new understanding of female sex steroid physiology [20]. 
Pelletier et al. in animal studies showed a relatively strong stimulating effect of DHEA on the density of vaginal nerve fibers. This may explain the beneficial effects of vaginal DHEA on sexual dysfunction observed in postmenopausal women [21].

\section{Case report}

A 50-year-old patient came in due to hyperkeratotic changes in the vulva and severe local symptoms of pruritus and pain for 3 years. During this period, the patient was treated several times (cream with estrogens, antifungal and antibacterial creams, and glucocorticosteroid creams) without a lasting effect. The last menstruation occurred in the patient at the age of 48 . The menopausal symptoms were of moderate intensity, mainly in the form of mild insomnia and hot flushes two or three times a day. Gynecological interviews: menarche occurred at the age of twelve, menstrual cycles were regular every twenty-eight days, normal menstrual bleeding lasted four days, two physiological deliveries. The patient is not treated due to chronic diseases. No risk factors for cancer, metabolic diseases and thromboembolic diseases. During the period of menopause, the patient's mother was diagnosed with lichen sclerosus atrophic (LSA) with severe pruritus. The patient's mother was treated topically with vitamin $A$ ointments, corticosteroid ointments, and ointments with estrogens with little satisfactory and short-term effect. In the patient, pruritus and pain in the vulva increased in the summer, hindering normal functioning. Current Pap test - normal result. In a gynecological examination, the vulva is closed, atrophic changes with focal hyperkeratosis on the entire surface of the labia. Normal perineum, vagina is narrow and short. Vaginal mucosa, pale without secretion, inelastic. Vaginal part of uteri cervix is cylindrical, the external orifice is normal. Uterus anteverted, small, smooth, without pathological resistance in the projection of the ovaries. Vaginal part of uteri cervix normal in the specula. Vaginal ultrasound examination: Uterus anteverted, dimensions: $45 \times 43 \mathrm{~mm}$. Homogeneous echostructure of the uterus. Endometrium thickness $3.2 \mathrm{~mm}$. Cervix dimensions: $28 \times 22 \mathrm{~mm}$. Ovaries typically located: right $20 \times 18 \mathrm{~mm}$, left $21 \times 11 \mathrm{~mm}$, without visible ovarian follicles and pathological changes. Mammographic examination was normal. The patient was referred for vulvoscopy. The result - mixed vulvar dermatosis with a high component of hyperkeratosis, layer of epidermis on the right side $1 / 3$ upper right labia and 1/2 length of the left labia, biopsy the above-mentioned of the vulva area is indicated. A vulva biopsy was performed from the indicated areas. Histopathological examination results: left lip $1 / 2$ length and left labia, upper $1 / 3-$ fragments of skin with appendages and chronic inflam- mation. The patient was offered topical treatment with estrogens or DHEA vaginal globules for consideration. The patient decided to use DHEA globules in view of previous unsuccessful attempts at estrogen treatment. After discussing the use of the drug and possible side effects, she was administered prasterone $6.5 \mathrm{mg}$ daily in the form of vaginal globules. The patient was informed about the possibility of daily contact by phone in case of adverse effects. An appointment is scheduled in four weeks. The patient returned four weeks later, reporting a significant reduction in itching and pain relief. Changes in a gynecological examination - significant reduction of atrophic changes with focal hyperkeratosis over the entire surface of the labia, vaginal mucosa pale-pink with secretion, more elastic. Due to the objective and subjective improvement, the patient asked to continue the therapy. Currently, the patient is under observation.

\section{Conclusions}

Based on the literature data and our own experience, it can be concluded that the use of vaginal DHEA in menopausal patients with symptoms of vaginal atrophy is an effective and safe method of treatment.

\section{Disclosure}

The authors report no conflict of interest.

\section{References}

1. Bachmann GA, Leiblum SR. The impact of hormones on menopausal sexuality: a literature review. Menopause 2004; 11: 120-130.

2. Ulrich $\mathrm{L}$. The role of local vaginal estrogen treatment in urogenital atrophy. Exp Rev Obstet Gynecol 2012; 7: 545-556.

3. Kagan R, Williams RS, Pan K, et al. A randomized, placebo- and activecontrolled trial of bazedoxifene/conjugated estrogens for treatment of moderate to severe vulvar/vaginal atrophy in postmenopausal women. Menopause 2010; 17: 281-289.

4. Di Donato V, Schiavi CM, lacobelli V, et al. Ospemifene for the treatment of vulvar and vaginal atrophy: A meta-analysis of randomized trials. Part I: Evaluation of efficacy. Maturitas 2019; 121: 86-92.

5. Pickar JH, MacNeil T, Ohleth K. SERMs: progress and future perspectives. Maturitas 2010; 67: 129-138.

6. Zohrabi I, Abedi P, Ansari S, et al. The effect of oxytocin vaginal gel onvaginal atrophy in postmenopausal women: a randomized controlled trial. BMC Womens Health 2020; 20: 108.

7. Le Donne M, Caruso C, Mancuso A, et al. The effect of vaginally administered genistein in comparison with hyaluronic acid on atrophic epithelium in postmenopause. Arch Gynecol Obstet 2011; 283: 1319-1323.

8. Li FG, Deans R, Nesbitt-Hawes E, et al. Fractionated Laser for Vaginal Atrophy Symptoms: A Randomized, Double-Blind Placebo Controlled Study. J Minim Invasive Gynecol 2020; 27: S69.

9. Sahu P, Gidwani B, Dhongade HJ. Pharmacological activities of dehydroepiandrosterone: A review. Steroids 2020; 153108507.

10. Greaves RF, Wudy SA, Badoer E, et al. A tale of two steroids: The importance of the androgens DHEA and DHEAS for early neurodevelopment. J Steroid Biochem Mol Biol 2019; 188: 77-85.

11. Panjari M, Davis SR. DHEA for postmenopausal women: A review of the evidence. Maturitas 2011; 70: 22-25. 
12. Traish AM, Kang HP, Saad F, Guay AT. Dehydroepiandrosterone (DHEA) - a precursor steroid or an active hormone in human physiology. J Sex Med 2011; 8: 2960-2982.

13. Calvo E, Luu-The V, Morissette J, et al. Pangenomic changes induced by DHEA in the skin of postmenopausal women. J Steroid Biochem Mol Biol 2008; 112: 186-193.

14. Labrie F, Bélanger A, Bélanger P, et al. Metabolism of DHEA in postmenopausal women following percutaneous administration. J Steroid Biochem Mol Biol 2007; 103: 178-188.

15. Leblanc M, Labrie C, Bélanger A, et al. Pharmacokinetics of oral dehydroepiandrosterone (DHEA) in the ovariectomised cynomolgus monkey. J Steroid Biochem Mol Biol 2002; 81: 159-164.

16. Labrie F. Effect of intravaginal DHEA on serum DHEA and eleven of its metabolitesin postmenopausal women. J Steroid Biochem Mol Bio 2008; 111: 178-194.

17. Archer D. Dehydroepiandrosterone intra vaginal administration for the management of postmenopausal vulvovaginal atrophy. J Steroid Biochem Mol Biol 2015; 145: 139-143.

18. Labrie F, Archer D, Bouchard C, et al. Intravaginal prasterone (DHEA), a highly efficient and the only physiological treatment of vaginal atrophy. Maturitas 2009; 63 (Suppl 1): S29.

19. Barton L, Shuster L, Dockter T, et al. Systemic and local effects of vaginal dehydroepiandrosterone (DHEA): NCCTG N10C1 (Alliance). Support Care Cancer 2018; 26: 1335-1343.

20. Labrie F, Archer D. Efficacy of intravaginal dehydroepiandrosterone. (DHEA) on moderate to severe dyspareunia and vaginal dryness, symptoms of vulvovaginal atrophy, and of the genitourinary syndrome of menopause. Menopause 2018; 25: 1339-1353.

21. Pelletier G, Ouellet J, Martel C, Labrie F. Effects of ovariectomy and dehydroepiandrosterone (DHEA) on vaginal wall thickness and innervation. J Sex Med 2012; 9: 2525-2533. 